\title{
Dauerhafte Androgendeprivation mit Histrelin
}

- Im metastasierten Stadium des Prostatakarzinoms ist die Testosterondeprivation die wichtigste palliative Therapieoption, sofern es sich um hormonsensitive Tumoren handelt. Die früher durchgeführte chirurgische Kastration wird heute meist durch eine Behandlung mit dem GonadotropinReleasing Hormon (GnRH-)-Agonisten (auch LHRH-Agonisten) ersetzt. Mit Histrelin steht ein stark potenter Wirkstoff in Form eines Jahresimplantats $\left(\right.$ Vantas $^{\circledR}$ ) zur Verfügung, das jeweils über ein Jahr kontinuierlich niedrige Testosteronspiegel erzeugt. Darauf verwiesen der Vorsitzende des IQUO Dr. Götz Geiges, Berlin, Prof. Dr. Kurt Miller, Charité Berlin, und Dr. Matthias Schulze, Markkleeberg, im Rahmen des 2. IQUO-Kongresses (Interessenverband zur Qualitätssicherung der Arbeit niedergelassener Uro-Onkologen in Deutschland e.V.).
Nach einer kurzen agonistischen Wirkphase verringert sich infolge der dauerhaften Stimulierung durch synthetische GnRH-Analoga die LHRH-Rezeptordichte in der Hypophyse. Es wird weniger luteinisierendes Hormon ausgeschüttet, damit verbunden geht die testikuläre Androgenproduktion zurück. Besonders zuverlässig gelingt dies mit Histrelin. Der Wirkstoff ist deutlich potenter als andere LHRH-Agonisten. Das Implantat wird subkutan an der Innenseite des Oberarms eingesetzt und setzt zwölf Monate gleichmäßig $50 \mu \mathrm{g} / \mathrm{d}$ Histrelin frei. Testosteron-Durchbrüche wie unter anderen LHRHAnaloga lassen sich so vermeiden. Vier bis sechs Wochen nach Einsetzen des Implantats sinken die Serumtestosteronwerte dauerhaft unter 20 ng/dl und damit auf Kastrationsniveau. Wird das Implantat entfernt, normalisiert sich der Testosteronspiegel meist inner- halb eines Monats. Damit gehen auch die bei Hormonsuppression üblichen Nebenwirkungen wie Hitzewallungen zurück.

Nach einem Jahr wird das Implantat ausgetauscht oder entfernt. An der Implantationsstelle kann es zu einer lokalen Reaktion kommen, die in den meisten Fällen mild ausfällt und innerhalb von zwei Wochen abklingt. In einer neuen Phase IV-Studie, die im April dieses Jahres angelaufen ist, wird der Nutzen von Histrelin im Praxisalltag untersucht. Die View-Studie mit einer Beobachtungszeit von 15 Monaten konzentriert sich auf den Krankheitsverlauf (Entwicklung der PSA-Werte und Testosteronspiegel) und die Lebensqualität unter der Therapie. Noch bis Ende März 2013 können IQUO-Mitglieder Patienten in die ViewStudie einschließen. Kathrin von Kieseritzky

Brunchsymposium „Prostatakarzinom: Eine praxisnahe Betrachtung der Hormontherapie", 2. IQUO-Kongress, Berlin, 30. Juni 2012 Veranstalter: Orion Pharma, Hamburg

\section{Metastasiertes Nierenzellkarzinom: Therapieansprechen korreliert mit Gesamtüberleben}

- Tyrosinkinase-Inhibitoren (TKI) haben beim metastasierten Nierenzellkarzinom ( $m R C C)$ das Überleben der Patienten verlängert. Heute stehen neben Sunitinib (Sutent ${ }^{\oplus}$ )

Neue Info-Plattform für Ärzte

- Auf der Wissenschaftsplattform www. androgen-arena.de finden Ärzte vielseitige und praxisbezogene Informationen zur Behandlung des Hypogonadismus, vor allem des Altershypogonadismus. Neben Fakten zum klinischen Bild und zur Therapie gibt es auch Trendthemen wie zum Beispiel „IGeL-Strategien und -Tipps für die Praxis", die in der "Pro \& Contra"Box von allen Seiten beleuchtet werden. In der Rubrik Fallbeispiele können Kollegen interaktiv Erfahrungen am konkreten Fall austauschen.

Nach Informationen von Ferring, Kiel weitere TKI in der mRCC-Erstlinie zur Verfügung. Mit welchem Medikament aber sollte die Therapie begonnen werden? Daten von der diesjährigen Jahrestagung der American Society of Clinical Oncology (ASCO), liefern Hinweise dazu. „In unserer Untersuchung korrelierte das Ansprechen auf einen TKI in der mRCC-Erstlinientherapie mit dem Gesamtüberleben", sagte PD Dr. Viktor Grünwald, Hannover, auf einem Post-ASCO-Pressegespräch von Pfizer Oncology Deutschland (21.6.2012, Berlin). Daher ist das Ansprechen ein wichtiger Wirksamkeitsparameter und ein relevantes Kriterium für die Therapieentscheidung.

Die wichtige Rolle von TKI wie Sunitinib in der mRCC-Erstlinientherapie untermauerte Grünwald mit Daten einer von ihm auf dem ASCO vorgestellten Korrelationsanalyse. Hierbei ergab sich eine positive Korrelation zwischen dem Ansprechen beziehungsweise der Tumorschrumpfung und dem Gesamtüberleben [Grünwald V et al. ASCO 2012 Poster, abstr. 4631].,Das Ansprechen, gemessen an derTumorschrumpfung, war in den uni- und multivarianten Analysen ein unabhängiger prognostischer Marker für das Behandlungs- ergebnis", so Grünwald. Gestützt wird diese Einschätzung durch die Ergebnisse einer weiteren auf dem ASCO präsentierten retrospektiven Analyse, die den Einfluss des Ansprechens unter Sunitinib auf die Prognose untersuchte. Molina et al. poolten hierzu Daten von sechs Phase-II/III-Studien mit insgesamt 1.059 Patienten, von denen drei Viertel in der Erstlinie, die übrigen in der Zweitlinie behandelt worden waren. Nach den RECIST-Kriterien sprachen 398 Patienten mit einer kompletten oder partiellen Remission auf die Sunitinib-Therapie an. Gegenüber den Non-Respondern erreichten sie ein signifikant längeres medianes OS (40,1 vs. 14,5 Monate) [Molina AM et al. ASCO 2012, Poster, abstr. 4542]. Die Analyse ergab zudem, dass die Ansprechrate unter Sunitinib mit der Therapiedauer zunimmt. Nach sechs Wochen sprachen $26 \%$ der Responder an, nach 24 Wochen $86 \%$. Grünwald erklärte, dass diese Daten auch ein wichtiger Beleg dafür seien, die Sunitinib-Therapie nicht zu früh abzubrechen, da ein Ansprechen häufig auch noch später erfolgen kann.

Nach Informationen von Pfizer, Berlin 